\title{
Non-unitary Scattering and Capture. I. Hilbert Space Theory
}

\author{
E. B. Davies \\ St. John's College, Oxford OX13JP, UK
}

\begin{abstract}
By carrying out a general analysis of properties of the wave operators for the non-unitary scattering theory which arises in connection with the use of complex "optical" potentials in nuclear scattering and elsewhere, we clarify some puzzling differences between two recent approaches to this subject.
\end{abstract}

\section{Introduction}

In two recent papers $[1,12] \mathrm{B}$. Simon and the author have discussed scattering problems for one-parameter contraction semigroups on Hilbert space. These semigroups arise in the complex "optical" potential approach to scattering theory of multiparticle systems, in which the effect of "external" channels is taken into account in a phenomenological manner by including absorptive or decay terms in the interaction. The virtue of the model is that it eliminates a large number of degrees of freedom and hence leaves one with a more analytically tractable problem.

Another virtue of this approach is that one may easily incorporate into it effects of interactions with the electromagnetic field. In standard multiparticle scattering theory such common phenomena as the capture of a neutron by a nucleus cannot occur, because the compound nucleus which is formed is unstable, and only becomes stable upon the emission of $\gamma$-radiation. On the other hand we shall show in [2] that in an appropriate quantum dynamical semigroup model of neutron scattering capture may indeed occur.

We start by assuming that the free evolution is described by a self-adjoint Hamiltonian $H_{0}$ on the Hilbert space $\mathscr{H}$, and that the interaction Hamiltonian is $H=H_{0}+V$, where the perturbation $V$ is not self-adjoint. The following is the first of a series of hypotheses, each of which will be assumed to hold after its formulation, without further mention.

Hypothesis $A$. The Hamiltonian $H_{0}$ is semi-bounded. Also $V=V_{1}-i V_{0}$ where the self-adjoint operators $V_{0}$ and $V_{1}$ have $\operatorname{Dom}\left(V_{i}\right) \supseteqq \operatorname{Dom}\left(H_{0}\right)$, and $V_{0} \geqq 0$. Finally, $V_{i}$ are relatively compact with respect to $H_{0}$. 
It is an immediate consequence of this hypothesis that $V$ has relative bound zero with respect to $H_{0}$, and that $-i H$ is the generator of a strongly continuous one-parameter contraction semigroup on $\mathscr{H},[7$, p. 499].

We define a bound state of $H$ to be an eigenvector with real eigenvalue, and denote by $\mathscr{H}_{b}$ the closed linear span of the bound states. According to [10, p. 113] the essential spectrum of $H$ is equal to that of $H_{0}$ so the non-real spectrum consists of a sequence of eigenvalues $\lambda_{n}$ whose only possible limit points are in $\operatorname{Sp}\left(H_{0}\right) \subseteq \mathbb{R}$. Associated with each $\lambda_{n}$ is a generally non-orthogonal finite rank spectral projection $P_{n}$ of $H$; the restriction of $H$ to $P_{n} \mathscr{H}$ has spectrum $\left\{\lambda_{n}\right\}$ but a possibly non-trivial Jordan form. The point spectral subspace $\mathscr{H}_{p}$ of $H$ is defined to be the closed linear span of $P_{n} \mathscr{H}$ for all $\lambda_{n}$ with $\operatorname{Im}\left(\lambda_{n}\right)<0$.

There is yet another subspace of $\mathscr{H}$ invariant under $e^{-i H t}$, namely the subspace of decaying states

$$
\mathscr{H}_{d}=\left\{\phi \in \mathscr{H}: \lim _{t \rightarrow \infty}\left\|e^{-i H t} \phi\right\|=0\right\} .
$$

It is easy to show that $\mathscr{H}_{p} \cong \mathscr{H}_{d}$ and one would like to be able to find conditions under which $\mathscr{H}_{p}=\mathscr{H}_{d}$. This is an open problem.

Lemma 1. If $\phi \in \operatorname{Dom}(H)$ and $H \phi=\lambda \phi$ for some real $\lambda$ then $V_{0} \phi=0$ and $\left(H_{0}+V_{1}\right) \phi=\lambda \phi$. Moreover $\mathscr{H}_{b} \perp \mathscr{H}_{d}$ and

$$
\begin{aligned}
& e^{-i H t} \mathscr{H}_{b}=\mathscr{H}_{b}, \quad e^{-i H t} \mathscr{H}_{b}^{\perp} \subseteq \mathscr{H}_{b}^{\perp} \\
& e^{-i H t} \mathscr{H} \subseteq \mathscr{H}
\end{aligned}
$$

for all $t \geqq 0$.

Proof. For any bound state $\phi$

$$
\lambda\|\phi\|^{2}=\left\langle\left(H_{0}+V_{1}\right) \phi, \phi\right\rangle-i\left\langle V_{0} \phi, \phi\right\rangle
$$

so $\left\langle V_{0} \phi, \phi\right\rangle=0$. Since $V_{0} \geqq 0$ it follows that $V_{0} \phi=0$. Hence $H, H^{*}$ and $\left(H_{0}+V_{1}\right)$ coincide on $\mathscr{H}_{b}$. The fact that the restriction of $e^{-i H t}$ to $\mathscr{H}_{b}$ is a one-parameter unitary group follows immediately. To prove that $\mathscr{H}_{b}^{\perp}$ is invariant under $e^{-i H t}$ we note as in [12] that if $\psi \in \mathscr{H}_{b}^{\perp}$ and $\phi$ is a bound state of $H$ then

$$
\left\langle e^{-i H t} \psi, \phi\right\rangle=\left\langle\psi, e^{i H^{*} t} \phi\right\rangle=e^{-i \lambda t}\langle\psi, \phi\rangle=0 .
$$

If $\psi \in \mathscr{H}_{d}$ and $H \phi=\lambda \phi$ with $\lambda \in \mathbb{R}$ then

$$
\begin{aligned}
0 & =\lim _{t \rightarrow \infty}\left\langle e^{-i H t} \psi, \phi\right\rangle \\
& =\lim _{t \rightarrow \infty}\left\langle\psi, e^{i H^{*} t} \phi\right\rangle \\
& =\lim _{t \rightarrow \infty} e^{-i \lambda t}\langle\psi, \phi\rangle .
\end{aligned}
$$

Therefore $\langle\psi, \phi\rangle=0$, and $\mathscr{H}_{d} \perp \mathscr{H}_{b}$. The invariance of $\mathscr{H}_{d}$ is elementary.

As in ordinary scattering theory the wave operators are defined by

$$
\begin{aligned}
& W_{-} \phi=\lim _{t \rightarrow \infty} e^{-i H t} e^{i H_{0} t} \phi \\
& W_{+} \phi=\lim _{t \rightarrow \infty} e^{i H_{0} t} e^{-i H t} \phi
\end{aligned}
$$


their domains being the sets of $\phi$ for which the limits exist. The extent to which the domains and ranges of $W_{ \pm}$can be interpreted as in the unitary case is limited by the fact that $e^{-i H t}$ is only defined for $t \geqq 0$. We handle $W_{-}$using Cook's method.

Hypothesis $B$. The Hamiltonian $H_{0}$ has purely absolutely continuous spectrum. Moreover there is a dense subspace $\mathscr{D}$ of $\mathscr{H}$ such that

$$
\int_{0}^{\infty}\left\|V e^{i H_{0} t} \phi\right\| d t<\infty
$$

for all $\phi \in \mathscr{D}$.

Before starting the next lemma we recall from [1] that the absolutely continuous subspace $\mathscr{H}_{a c}$ for $H$ is defined to be the closure of

$$
M(H)=\left\{\phi: \int_{0}^{\infty}\left|\left\langle e^{-i H t} \phi, \psi\right\rangle\right|^{2} d t \leqq c_{\phi}\|\psi\|^{2} \text { for all } \psi \in \mathscr{H}\right\} .
$$

It is elementary that $\mathscr{H}_{a c}$ is invariant under $e^{-i H t}$ and that

$$
\mathscr{H}_{p} \cong \mathscr{H}_{a c} \subseteq \mathscr{H}_{b}^{\perp} .
$$

Lemma 2. The limit $W_{-} \phi$ exists for all $\phi \in \mathscr{H}$ and defines a one-one contraction $W_{-}$from $\mathscr{H}$ into $\mathscr{H}_{\text {ac }}$.

Proof. We refer to [1] for the proof that $W_{-}$is a contraction from $\mathscr{H}$ into $\mathscr{H}_{a c}$ and give Martin's proof [8] that $W_{-}$is one-one. Since

$$
e^{-i H_{t}} W_{-} \phi=W_{-} e^{-i H_{0} t} \phi
$$

for all $\phi \in \mathscr{H}$ and all $t \geqq 0$, the subspace

$$
\mathscr{L}=\left\{\phi \in \mathscr{H}: W_{-} \phi=0\right\}
$$

satisfies

$$
e^{-i H_{0} t} \mathscr{L} \leqq \mathscr{L}
$$

for all $t \geqq 0$. Since $H_{0}$ is semibounded (2) also holds for all $t \leqq 0$ by analytic continuation in $t$. But if $\phi \in \mathscr{D}$ then

$$
W_{-} \phi=\phi-i \int_{0}^{\infty} e^{-i H s} V e^{i H_{0} s} \phi d s
$$

so

$$
\begin{aligned}
\lim _{t \rightarrow \infty} & \left\|W_{-} e^{i H_{0} t} \phi\right\| \\
& =\lim _{t \rightarrow \infty}\left\|e^{i H_{0} t} \phi-i \int_{t}^{\infty} e^{-i H(s-t)} V e^{i H_{0} s} \phi d s\right\| \\
& =\|\phi\| .
\end{aligned}
$$

By density arguments we conclude that

$$
\lim _{t \rightarrow \infty}\left\|W_{-} e^{i H_{0} t} \phi\right\|=\|\phi\|
$$

for all $\phi \in \mathscr{H}$. Combining this with (2) we obtain $\mathscr{L}=0$. 


\section{Domain of the Wave Operator $W_{+}$}

The proof of the existence of the limits, $W_{+} \phi$ is not easy. In [1] the author used the Kato-Birman theory to show that $\operatorname{Dom}\left(W_{+}\right) \supseteqq \mathscr{H}_{a c}$, while in [2] Simon adapted the Enss theory to show that $\operatorname{Dom}\left(W_{+}\right)=\mathscr{H}_{b}^{\perp}$. The two papers are not directly comparable because the Kato-Birman theory depends on abstract operator hypotheses while the Enss theory uses geometrical ideas, but for potential scattering problems the Enss theory is more powerful except possibly in the spherically symmetric case. It is pleasing therefore that we can show under simple general conditions that $\mathscr{H}_{a c}=\mathscr{H}_{b}^{\perp}$, so that the two approaches give qualitatively identical conclusions.

We start by pointing out that if $H_{1}=H_{0}+V_{1}$, the scattering between $H_{0}$ and $H$ can be split into two independent problems between $H_{0}$ and $H_{1}$, and between $H_{1}$ and $H$. We assume that the first problem is amenable to one of the standard methods of analysis [9], and in particular assume the following.

Hypothesis $C$. The Hamiltonian $H_{1}$ may have non-empty point spectrum, but it has empty singular continuous spectrum.

By the theory of unitary dilations of one parameter contraction semigroups $[3,13]$ there is an orthogonal decomposition

$$
\mathscr{H}=\mathscr{H}_{u} \oplus \mathscr{H}_{\text {cnu }}
$$

such that each of the subspaces is invariant under $e^{-i H t}$ for all $t \geqq 0$, and $\mathscr{H}_{u}$ is the largest subspace on which $e^{-i H t}$ are unitary operators. The semigroup is said to be completely non-unitary on $\mathscr{H}_{c n u}$.

Lemma 3. The subspace $\mathscr{H}_{u}$ is invariant under $e^{-i H_{1} t}$ for all $t \in \mathbb{R}$ and

$$
e^{-i H t} \phi=e^{-i H_{1} t} \phi
$$

for all $\phi \in \mathscr{H}_{u}$ and all $t \geqq 0$.

Proof. If $\phi \in \mathscr{H}_{u} \cap \operatorname{Dom}(H)$ and we put $\phi_{t}=e^{-i H t} \phi$ then

$$
0=\frac{\partial}{\partial t}\left\|\phi_{t}\right\|^{2}=-2\left\langle V_{0}, \phi_{t}, \phi_{t}\right\rangle
$$

Therefore $V_{0} \phi_{t}=0$ so

$$
\frac{\partial \phi_{t}}{\partial t}=-i H_{1} \phi_{t}
$$

whose solution is $\phi_{t}=e^{-i H_{1} t} \phi$. Since $\mathscr{H}_{u} \cap \operatorname{Dom}(H)$ is dense in $\mathscr{H}_{u}$, (3) holds for all $\phi \in \mathscr{H}_{u}$. The invariance of $\mathscr{H}_{u}$ under $e^{-i H_{1} t}$ for $t \geqq 0$ is immediate. But $V_{1}$ is relatively compact with respect to $H_{0}$, so $H_{1}$ is semibounded and the invariance of $\mathscr{H}_{u}$ under $e^{-i H_{1} t}$ for $t \leqq 0$ can be proved by analytic continuation in $t$.

Lemma 4. If $e^{B t}$ is a completely non-unitary one-parameter contraction semigroup on $\mathscr{H}$ then

$$
\mathscr{H}_{a c}(B)=\mathscr{H} .
$$


Proof. We first consider the case of a completely non-unitary one-parameter semigroup of isometries. By $[3 ; 13$, p. 151] there is an isomorphism of $\mathscr{H}$ onto $L^{2}\{(0, \infty) ; \mathscr{N}\}$ for a suitable auxiliary space $\mathscr{N}$ such that

$$
\left(e^{B t} f\right)(x)=\left\{\begin{array}{lll}
f(x-t) & \text { if } & x \geqq t \geqq 0 \\
0 & \text { if } & 0 \leqq x<t
\end{array}\right.
$$

for all $f \in L^{2}$ and $t \geqq 0$. If $\mathscr{D}$ is the subspace of $C^{\infty} \mathscr{N}$-valued functions with compact support in $(0, \infty)$ then $\mathscr{D} \cong M(B)$. Since $\mathscr{D}$ is dense in $\mathscr{H}$ the result follows.

In the general case let $e^{-i K t}$ denote the minimal unitary dilation on $\mathscr{K} \supseteqq \mathscr{H}$ of $e^{B t}$, so that $K$ has purely absolutely continuous spectrum by $[3 ; 13, \mathrm{p} .84]$. If we define

$$
\mathscr{L}=\widetilde{\operatorname{lin}}\left\{e^{-i K t} \mathscr{H}: t \leqq 0\right\}
$$

then $e^{-i K t} \mathscr{L} \subseteq \mathscr{L}$ for all $t \leqq 0$ and $\mathscr{H} \leqq \mathscr{L}$. If $s, t \geqq 0$ and $f, g \in \mathscr{H}$ and $P$ is the projection of $\mathscr{K}$ onto $\mathscr{H}$ then

$$
\begin{aligned}
\left\langle(1-P) e^{-i K t} f, e^{i K s} g\right\rangle \\
\quad=\left\langle P e^{-i K s}(1-P) e^{-i K t} f, g\right\rangle \\
=\left\langle P e^{-i K(s+t)} f, g\right\rangle-\left\langle P e^{-i K s} P e^{-i K t} f, g\right\rangle \\
=\left\langle e^{B(s+t)} f, g\right\rangle-\left\langle e^{B s} e^{B t} f, g\right\rangle \\
=0
\end{aligned}
$$

so

$$
(1-P) e^{-i K t} f \in \mathscr{L}^{\perp}
$$

Hence if we define $\mathscr{M}=\mathscr{H} \oplus \mathscr{L}^{\perp}$ then

$$
e^{-i K t} \mathscr{M} \subseteq \mathscr{M}
$$

for all $t \geqq 0$. The restriction $e^{C t}$ of $e^{-i K t}$ to $\mathscr{M}$ is a one-parameter semigroup of isometries. Since it is the sum of a completely non-unitary one parameter semigroup of isometries, to which we can apply the first part of the proof, and a one-parameter unitary group which has absolutely continuous spectrum because it is a restriction of $e^{-i K t}, M(C)$ is dense in $\mathscr{M}$.

If $f \in M(C)$ and $g \in \mathscr{H}$ then

$$
\begin{aligned}
& \int_{0}^{\infty}\left|\left\langle e^{B t} P f, g\right\rangle\right|^{2} d t \\
& =\int_{0}^{\infty}\left|\left\langle P e^{C t} P f, g\right\rangle\right|^{2} d t \\
& =\int_{0}^{\infty}\left|\left\langle e^{C t} f, g\right\rangle\right|^{2} d t \\
& \leqq c\|g\|^{2} .
\end{aligned}
$$

Hence $P f \in M(B)$, so $M(B)$ is dense in $\mathscr{H}$ as required. 
Theorem 5. Under Hypotheses $A, B, C$ we have

$$
\mathscr{H}_{a c}=\mathscr{H}_{b}^{\perp} \text {. }
$$

Proof. Since $H$ coincides with $H_{1}$ on $\mathscr{H}_{u}$ and $H_{1}$ has no singular continuous spectrum, there is an orthogonal direct sum decomposition

$$
\mathscr{H}_{u}=\mathscr{H}_{u, b} \oplus \mathscr{H}_{u, a c}
$$

where $\mathscr{H}_{u, b}$ is the closed linear span of those eigenstates of $H_{1}$ lying in $\mathscr{H}_{u}$, and actually equals $\mathscr{H}_{b}$. If $\phi \in \mathscr{H}_{u, a c}$ then $\phi \in \mathscr{H}_{a c}\left(H_{1}\right)$ so $\phi \in \mathscr{H}_{a c}(H)$. Applying Lemma 4 to the restriction of $e^{-i t H}$ to $\mathscr{H}_{c n u}$ we obtain

$$
\mathscr{H}_{a c} \supseteqq \mathscr{H}_{c n u} \oplus \mathscr{H}_{u, a c} \text {. }
$$

The proof is completed by reference to (1).

In the light of the above theorem and $[1,12]$ it is now reasonable to assume Hypothesis $D$. The limit $W_{+} \phi$ exists for all $\phi \in \mathscr{H}_{b}^{\perp}$.

\section{Invertibility of the Wave Operators}

In order to make further progress we need the following hypothesis, which is frequently satisfied but whose necessity is uncertain.

Hypothesis $E$. There is a conjugation operator $J$ on $\mathscr{H}$ such that for $i=0,1$

$$
J H_{0}=H_{0} J, \quad J V_{i}=V_{i} J .
$$

Lemma 6. The reverse wave operators

$$
V_{+} \phi=\lim _{t \rightarrow \infty} e^{i H^{*} t} e^{-i H_{0} t} \phi
$$

and

$$
V_{-} \phi=\lim _{t \rightarrow \infty} e^{-i H_{0} t} e^{i H^{*} t} \phi
$$

exist with domains

$$
\operatorname{Dom}\left(V_{+}\right)=\mathscr{H}, \quad \operatorname{Dom}\left(V_{-}\right)=\mathscr{H}_{b}^{\perp} .
$$

Moreover if $W_{+}$and $V_{-}$are extended to the whole of $\mathscr{H}$ by putting

$$
W_{+} \phi=V_{-} \phi=0
$$

for all $\phi \in \mathscr{H}_{b}$ then

$$
J W_{-} J=V_{+}=W_{+}^{*}=J V_{-}^{*} J .
$$

Proof. If $\phi \in \mathscr{H}$ then

$$
\begin{aligned}
V_{+} \phi & =\lim _{t \rightarrow \infty} e^{i H^{k} t} e^{-i H_{0} t} \phi \\
& =\lim _{t \rightarrow \infty} J e^{-i H t} e^{i H_{0} t} J \phi \\
& =J W_{-} J \phi .
\end{aligned}
$$


The proof of the existence of $V_{-}$is similar, using the fact that $J \mathscr{H}_{b}=\mathscr{H}_{b}$.

From their definitions it is evident that

$$
\left\langle W_{+} \phi, \psi\right\rangle=\left\langle\phi, V_{+} \psi\right\rangle
$$

for all $\phi \in \mathscr{H}_{b}^{\perp}$ and $\psi \in \mathscr{H}$. If $\phi \in \mathscr{H}_{b}$ then the left hand side vanishes by the definition of $W_{+} \phi$. Also

$$
V_{+} \psi \in \mathscr{H}_{a c}\left(H^{*}\right)=\mathscr{H}_{b}^{\perp}
$$

so $\left\langle\phi, V_{+} \psi\right\rangle=0$. Hence (4) holds for all $\phi, \psi \in \mathscr{H}$ and $V_{+}=W_{+}^{*}$.

The conditions of the following theorem are certainly not always satisfied, but when they are the spectral analysis of $H$ is considerably simplified.

Theorem 7. The following conditions are equivalent:

(i) The range of $W_{-}$is a closed subspace;

(ii) The scattering operator $S=W_{+} W_{-}$maps $\mathscr{H}$ one-one onto $\mathscr{H}$;

(iii) One has a Banach space direct sum decomposition

$$
\mathscr{H}=\left(\text { Range } W_{-}\right) \oplus \mathscr{H}_{d} \oplus \mathscr{H}_{b} .
$$

The first two subspaces need not be orthogonal to each other but both are orthogonal to $\mathscr{H}_{b}$.

Proof. If (ii) holds then by the inverse mapping theorem there extends an $\alpha>0$ such that $\|S \phi\| \geqq \alpha\|\phi\|$ for all $\phi \in \mathscr{H}$. It follows that $\left\|W_{-} \phi\right\| \geqq \alpha\|\phi\|$ for all $\phi \in \mathscr{H}$ and this implies (i).

Conversely if $W_{-}$has closed range then there is a constant $\alpha>0$ such that $\left\|W_{-} \phi\right\| \geqq \alpha\|\phi\|$ for all $\phi \in \mathscr{H}$. Now

$$
\begin{aligned}
\|S \phi\| & =\lim _{t \rightarrow \infty}\left\|e^{i H_{0} t} e^{-i H t} W_{-} \phi\right\| \\
& =\lim _{t \rightarrow \infty}\left\|e^{i H_{0} t} W_{-} e^{-i H_{0} t} \phi\right\| \\
& \geqq \alpha\left\|e^{-i H_{0} t} \phi\right\|=\alpha\|\phi\|
\end{aligned}
$$

so $S$ is one-one with closed range. But

$$
\begin{aligned}
\operatorname{Range}\left(V_{+}\right) & =\operatorname{Range}\left(J W_{-} J\right) \\
& =J \operatorname{Range}\left(W_{-}\right)
\end{aligned}
$$

is a closed subspace, so $V_{-} V_{+}$is one-one with closed range by a similar argument. Now

$$
V_{-} V_{+}=W_{-}^{*} W_{+}^{*}=S^{*} .
$$

If $\phi \perp$ Range $S$ then for all $\psi \in \mathscr{H}$

$$
0=\langle S \psi, \phi\rangle=\left\langle\psi, S^{*} \phi\right\rangle
$$

so $S^{*} \phi=0$ and $\phi=0$. Hence Range $S=\mathscr{H}$. 
Since (i) is implicit in (iii) we are left with proving (iii) from (i) and (ii). If $W_{-} \phi \in \mathscr{H}_{d}$ then

$$
\begin{aligned}
\|S \phi\| & =\lim _{t \rightarrow \infty}\left\|e^{i H_{0} t} e^{-i H t} W_{-} \phi\right\| \\
& =\lim _{t \rightarrow \infty}\left\|e^{-i H t} W_{-} \phi\right\|=0
\end{aligned}
$$

so $S \phi=0$ and $\phi=0$. This proves

(Range $\left.W_{-}\right) \cap \mathscr{H}_{d}=0$.

Moreover $\mathscr{H}_{b}$ is clearly orthogonal to both subspaces. If $\phi \in \mathscr{H}$ then $\phi=\phi_{a c}+\phi_{b}$ where $\phi_{a c} \in \mathscr{H}_{b}^{\perp}$ and $\phi_{b} \in \mathscr{H}_{b}$. Then $W_{+} \phi_{b}=0$ and

$$
W_{+} \phi_{a c}=\lim _{t \rightarrow \infty} e^{i H_{0} t} e^{-i H t} \phi_{a c}=\psi \text { (say). }
$$

Since $S$ has range equal to $\mathscr{H}$ there exists $\phi^{\prime} \in$ Range $W_{-}$with $W_{+} \phi^{\prime}=\psi$. Now putting $\phi^{\prime \prime}=\phi_{a c}-\phi^{\prime}$ we obtain

$$
\lim _{t \rightarrow \infty} e^{i H_{0} t} e^{-i H t} \phi^{\prime \prime}=0
$$

so $\phi^{\prime \prime} \in \mathscr{H}_{d}$. We thus have $\phi=\phi_{b}+\phi^{\prime}+\phi^{\prime \prime}$ where $\phi_{b} \in \mathscr{H}_{b}, \phi^{\prime} \in$ Range $W_{\text {_ }}$ and $\phi^{\prime \prime} \in \mathscr{H}_{d}$.

Hypothesis E is certainly not absolutely necessary. For example suppose that $\mathscr{H}=L^{2}\left(\mathbb{R}^{3}\right), H_{0}=-\frac{1}{2 m} \Delta$ and Hypotheses $\mathrm{A}-\mathrm{D}$ are satisfied.

Theorem 8. The conditions of Theorem 7 are still equivalent if Hypothesis $E$ is replaced by the condition that the operator $V$ is rotationally invariant.

Proof. The only change is in the proof that if $S$ is one-one with closed range then it is onto. Since $S$ commutes both with $H_{0}$ and with rotations, it is a multiplication operator in each angular momentum sector, and hence is normal, $S S^{*}=S^{*} S$. This implies the required result.

If the conditions of Theorem 10 are satisfied then the restriction of $H$ to Range $W_{\text {- }}$ is a spectral operator of scalar type $[4,5]$. Even if this fails it is still possible that for some finite interval $[a, b]$ the subspace $W_{-} \mathscr{H}_{[a, b]}$ is closed, where $\mathscr{H}_{[a, b]}$ is the spectral subspace of $H_{0}$ for the interval $[a, b]$. One may then show that $H$ is a spectral operator of the scalar type on $W_{-} \mathscr{H}_{[a, b]}$ and that $S$ maps $\mathscr{H}_{[a, b]}$ one-one onto $\mathscr{H}_{[a, b]}$.

The following result shows that the properties of Theorem 10 are stable under small perturbations.

Theorem 9. Let $H_{\lambda}=H_{0}+V_{1}-i \lambda V_{0}$ where $\lambda$ lies in the half-plane $\mathbb{H}=\{\lambda: \operatorname{Re} \lambda>0\}$, and where Hypotheses $A-E$ are satisfied for all $\lambda$. If $U_{n} \cong \mathbb{H}$ is the set of $\lambda$ for which $W_{-}(\lambda)$ is a Fredholm operator of index $n$, then $H$ has finite point spectrum with total multiplicity $n$ for all $\lambda \in U_{n}$. If $U_{\infty}$ is the set of $\lambda \in \mathbb{H}$ such that $W_{-}(\lambda)$ has closed range of codimension $\infty$, then $U_{\infty}, U_{0}, U_{1}, U_{2}, \ldots$ are disjoint open subsets of $\mathrm{H}$.

Proof. If $n<\infty$ and $\lambda \in U_{n}$ then every eigenvector of $H$ lies in $\mathscr{H}_{d} \oplus \mathscr{H}_{b}$, which by Theorem 7 has dimension equal to $n$. Since $\mathscr{H}_{d} \oplus \mathscr{H}_{b}$ is invariant with respect to 
$e^{-i H t}$ the algebraic multiplicity of the point spectrum of $H$ is exactly equal to $n$. Since $W_{-}(\lambda)$ are strong limits of the analytic family $e^{-i H_{\lambda} t} e^{i H_{0} t}$ as $t \rightarrow \infty, W_{-}(\lambda)$ is strongly and hence norm analytic in $\lambda$, and so norm continuous in $\lambda$ for $\lambda \in \mathbb{H}$. But the class of (semi) Fredholm operators of given index is open in the norm topology $[7$, p. 230].

We henceforth assume

Hypothesis $F$. The equivalent conditions of Theorem 7 are satisfied for the Hamiltonian $H$.

The direct sum decomposition of Theorem 7 is associated with certain nonorthogonal projections. Such projections are frequently inconvenient, particularly in connection with probabilistic interpretations of the equations, and it is useful to have the following result concerning the orthogonal projection $P$ with kernel $\mathscr{H}_{d} \oplus \mathscr{H}_{b}$.

Theorem 10. If $\phi \in \mathscr{H}_{b}^{\perp}$ then

$$
\lim _{t \rightarrow \infty}\left\|e^{-i H t} \phi-P e^{-i H t} \phi\right\|=0 .
$$

Proof. The result is obvious for $\phi \in \mathscr{H}_{d}$ so we assume $0 \neq \phi \in \operatorname{Range} W_{-}$. By (5)

$$
\lim _{t \rightarrow \infty}\left\|e^{-i H t} \phi\right\|=\beta>0
$$

the left-hand side being a decreasing function of $t$ since $e^{-i H t}$ is a contraction semigroup. Given $\varepsilon>0$ let $a$ be large enough so that

$$
\left\|e^{-i H a} \phi\right\|<\beta(1+\varepsilon)
$$

and write

$$
e^{-i H a} \phi=\phi_{0}+\phi_{1}
$$

where $\phi_{0} \in \mathscr{H}_{d}$ and $\phi_{1} \in \mathscr{H}_{d}^{\perp} \cap \mathscr{H}_{b}^{\perp}$. Then for all $t \geqq a$

$$
\begin{aligned}
& \left\|e^{-i H(t-a)} \phi_{0}\right\|+\left\|e^{-i H(t-a)} \phi_{1}\right\| \\
& \quad \geqq\left\|e^{-i H(t-a)}\left(\phi_{0}+\phi_{1}\right)\right\|=\left\|e^{-i H t} \phi\right\| \geqq \beta
\end{aligned}
$$

so

$$
\begin{aligned}
\left\|\phi_{1}\right\| & \geqq\left\|e^{-i H(t-a)} \phi_{1}\right\| \\
& \geqq \beta-\left\|e^{-i H(t-a)} \phi_{0}\right\| \\
& \rightarrow \beta \text { as } t \rightarrow \infty .
\end{aligned}
$$

Now

$$
\begin{aligned}
\beta^{2}+\left\|\phi_{0}\right\|^{2} & \leqq\left\|\phi_{1}\right\|^{2}+\left\|\phi_{0}\right\|^{2} \\
& =\left\|e^{-i H a} \phi\right\|^{2}<\beta^{2}(1+\varepsilon)^{2}
\end{aligned}
$$

so

$$
\left\|\phi_{0}\right\|<\beta\left\{2 \varepsilon+\varepsilon^{2}\right\}^{1 / 2} .
$$


This establishes that

$$
\lim _{t \rightarrow \infty}\left\|(1-P) e^{-i H t} \phi\right\|=0
$$

for all $\phi=$ Range $W_{\text {- }}$ and hence all $\phi \in \mathscr{H}_{b}^{\perp}$.

\section{Some Simple Examples}

An analysis of simple cases shows that the conditions of Theorem 7 are not always satisfied. It frequently happens that the union of the sets $U_{n}$ defined in Theorem 9 is dense in $\mathrm{H}$ and that its complement consists of a set of curves corresponding to values of $\lambda$ at which new eigenvalues appear in the spectrum of $H$. On these critical curves we see by Theorems 7 and 9 that $W_{-}(\lambda)$ does not have closed range.

To justify these assertions we first consider the case where $V$ is a rank one perturbation. We put $\mathscr{H}=L^{2}(\mathbb{R})$ and define

$$
H_{\lambda}=H_{0}-i \lambda|f\rangle\langle f|
$$

where $f \in \mathscr{H}, \operatorname{Re}(\lambda)>0$ and $H_{0}$ is defined by

$$
\left(H_{0} g\right)(x)=x g(x)
$$

with the obvious domain.

Since the scattering operator

$$
S_{\lambda}=\lim _{t \rightarrow \infty} e^{i H_{0} t} e^{-2 i H_{\lambda} t} e^{i H_{0} t}
$$

commutes with $H_{0}$ whenever it exists, it is a multiplication operator on $L^{2}(\mathbb{R})$. We shall prove existence only under the following condition.

Hypothesis $G$. The Fourier transform of the function $|f|^{2}$ lies in $L^{1}(\mathbb{R})$.

Lemma 11. If $f$ satisfies Hypothesis $G$, then $S_{\lambda}$ exists for all $\operatorname{Re}(\lambda)>0$ and

$$
\left(S_{\lambda} g\right)(x)=\frac{1+i \lambda h(x)}{1+\overline{i \lambda h(x)}} g(x)
$$

for all $g \in L^{2}(\mathbb{R})$, where $h(z)$ is defined for all $\operatorname{Im}(z)<0$ by

$$
h(z)=\int_{-\infty}^{\infty} \frac{|f(u)|^{2}}{z-u} d u
$$

and has continuous boundary values on the real axis.

Outline of proof. For sufficiently small $|\lambda|$ one may expand the right-hand side of (6) in powers of $\lambda$, prove convergence term by term and then identify the two sides of (7) term by term in the power series expansions. The existence of $S_{\lambda}$ and the validity of (7) for all $\operatorname{Re}(\lambda)>0$ then follows by analytic continuation, using the fact that all the operators concerned are contractions depending analytically on $\lambda$. 
Since $h(x)$ is a continuous function of $x$ vanishing at $\pm \infty$ and $\operatorname{Re}(\lambda)>0$ implies that

$$
1+i \hat{h(x)} \neq 0
$$

for all $x \in \mathbb{R}$, we see that $S_{\lambda}$ has closed range unless there exists $x \in \mathbb{R}$ such that $i \lambda h(x)=-1$. This demonstrates that

$$
\mathbb{H} \backslash\left\{\sum_{n=1}^{n=\infty} U_{n}\right\}=\left\{i h(x)^{-1}: x \in \mathbb{R}\right\} .
$$

We now turn to the eigenvalue problem. A trivial calculation shows that all eigenvalues $E$ of $H$ have strictly negative imaginary part. Moreover a number $E$ is an eigenvalue of $H$ if and only if

$$
1+i \lambda h(E)=0 .
$$

For each $\lambda$ this equation may have several solutions, each of which is locally an analytic function of $\lambda$. The critical curves on which $S_{\lambda}$ is not one-one are seen to coincide with the values of $\lambda$ for which one of these eigenvalues meets the real axis (and disappears).

As an explicit example we recommend the case

$$
|f(x)|^{2}=\left(1+x^{2}\right)^{-1}
$$

for which all the relevant quantities are explicitly computable.

A similar but more complicated analysis using the eigenfunction expansion method $[6,11]$ can be carried out for Schrodinger operators with complex central potentials, in each angular momentum sector. Note that in $[5$, p. 2397] the hypothesis that certain functions $A^{ \pm}(\lambda)$ do not vanish for $0 \leqq \lambda<\infty$ is equivalent to the conditions of our Theorem 7 , modulo technicalities.

\section{References}

1. Davis, E.B.: Two-channel Hamiltonians and the optical model of nuclear scattering. Ann. Inst. H. Poincaré 29A, 395-413 (1978)

2. Davies, E.B.: Non-unitary scattering and capture. II. Dynamical semigroup theory. To appear

3. Davies, E.B.: One-parameter semigroups. London, New York: Academic Press 1980

4. Dowson, H.R.: Spectral theory of linear operators. London Math. Soc. Monographs. London, New York: Academic Press 1978

5. Dunford, N., Schwartz, J.T. : Linear operators Part 3. Spectral operators. Wiley-Interscience, 1971

6. Goldstein, C.: Perturbation of non-self-adjoint operators. I. Arch. Rat. Mech. Anal. 37, 268-296 (1970); II. Arch. Rat. Mech. Anal. 42, 380-402 (1971)

7. Kato, T.: Perturbation theory for linear operators. 1st Edition. Berlin, Heidelberg, New York: Springer 1966

8. Martin, Ph. A.: Scattering theory with dissipative interactions and time decay. Nuovo Cimento 30B, 217-238 (1975)

9. Reed, M., Simon, B.: Methods of modern mathematical physics Vol. 3. London, New. York: Academic Press 1978

10. Reed, M., Simon, B.: Methods of modern mathematical physics Vol. 4. London, New York: Academic Press 1978 
11. Sergent, P., Coudray, C.: The inverse problem at fixed energy for finite range complex potentials. Ann. Inst. H. Poincaré 29A, 179-205 (1978)

12. Simon, B.: Phase space analysis of simple scattering systems: extensions of some work of Enss. Duke Math. J. 46, 119-168 (1979)

13. Sz.-Nagy, B., Foias, C.: Harmonic analysis of operators on Hilbert space. Amsterdam: NorthHolland 1970

Communicated by B. Simon

Received April 24, 1979; in revised form October 23, 1979 